\title{
Correction to: Good response to tolvaptan shortens hospitalization in patients with congestive heart failure
}

\author{
Tomohito Kogure $^{1} \cdot$ Kentaro Jujo $^{1} \cdot$ Kazuyuki Hamada $^{1} \cdot$ Katsumi Saito $^{2} \cdot$ Nobuhisa Hagiwara $^{1}$
}

Published online: 12 December 2017

๑) Springer Japan KK, part of Springer Nature 2017

\section{Correction to: Heart Vessels \\ https://doi.org/10.1007/s00380-017-1072-6}

In the original publication of the article, the values of blood urea nitrogen (BUN) and plasma renin activity (PRA) have been published incorrectly and the corrected values are as follows.

In abstract, the following sentence should read as, “... Multivariate analysis revealed that blood urea nitrogen (BUN) [cutoff: $34 \mathrm{mg} / \mathrm{dL}$, odds ratio (OR) 9.0, 95\% confidence interval (CI) 1.42-57.3, $p<0.01]$ and plasma renin activity (PRA) (cutoff $4.7 \mathrm{ng} / \mathrm{mL} / \mathrm{h}$, OR $6.1,95 \% \mathrm{CI}$ $1.01-36.4, p<0.01$ ) at baseline were independent predictors for tolvaptan responsiveness..."

Under the section "Multivariate analysis", the last sentence should read as "...The only variables related to tolvap$\tan$ responsiveness were BUN and PRA level (odds ratio [OR] 9.01, 95\% confidence interval [CI] 1.42-57.3; OR $6.06,95 \%$ CI 1.01-36.4, respectively)."

The original article was corrected.

The original article can be found online at https://doi. org/10.1007/s00380-017-1072-6.

Kentaro Jujo

juken1123@mac.com

1 Department of Cardiology, Tokyo Women's Medical University, 8-1 Kawadacho, Shinjuku-ku, Tokyo 162-0054, Japan

2 Department of Cardiology, Nishiarai Heart Center Hospital, Tokyo, Japan 